\title{
The happiness gap between transition and non-transition countries
}

\section{Economic progress coupled with political and institutional stability is needed to reduce unhappiness}

Keywords: happiness gap, subjective well-being, transition, institutions

\section{ELEVATOR PITCH}

Since 1989, post-communist countries have undergone profound changes in their political, economic, and social structures and institutions. Across a range of development outcomes-in terms of the speed and success of reformstransition is an "unhappy process." The "happiness gap," i.e. the difference in average happiness levels between the populations of transition and non-transition economies, is closing, but at a slower pace than the process of economic convergence. Economic growth, as the determinant of a country's collective well-being, has been superseded by measurements of institutional quality and social development.

\section{KEY FINDINGS}

\section{Pros}

๑ The happiness gap between transition and non-transition as well as advanced countries is gradually closing.

๑ Economic and political stability still have a stronger influence on life satisfaction than economic growth in transition countries.

๑ Key factors to improve individual and societal well-being include perceived legitimacy of institutions, good governance, and rule of law.

( High levels of social capital, in particular social trust, improve life satisfaction and happiness in the medium term, even in harsh economic conditions.

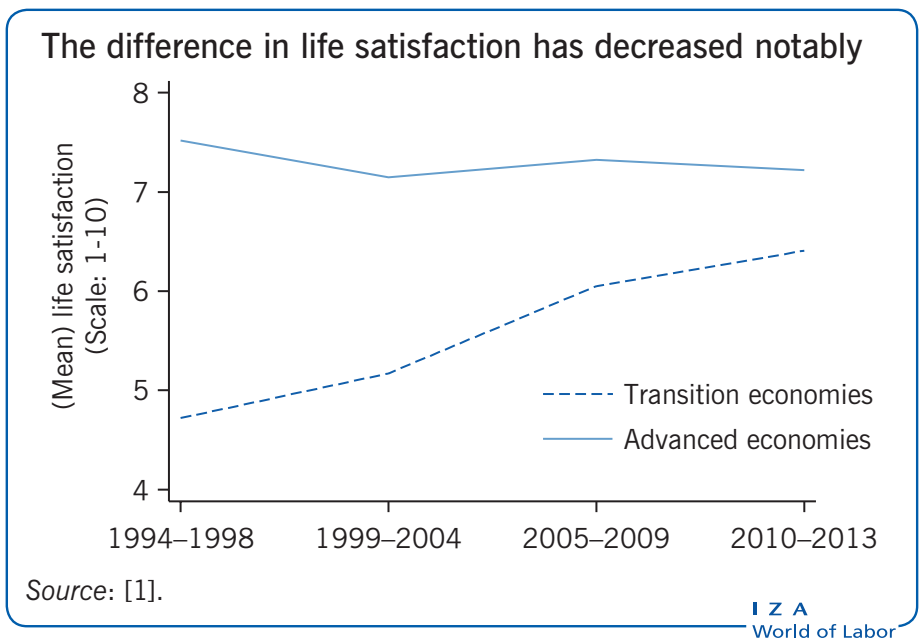

\section{Cons}

- The persistence of an "unconditional" happiness gap, regardless of economic progress, suggests a permanent "negative" transition effect.

- Economic convergence between transition and advanced countries may not be enough to close the happiness gap.

- Dissatisfaction with life may produce reform fatigue, thus threatening the stability of new and vulnerable economic and political institutions.

- Happiness gap estimates may be biased due to cultural differences across countries or generations.

\section{AUTHOR'S MAIN MESSAGE}

Raising income levels in post-communist countries has not compensated for the fall in well-being during the process of transition. Poor governance, corruption, and low levels of social capital are likely to further hinder both economic progress and the recovery of the populations' sense of life satisfaction. If transition countries are to achieve convergence with advanced economies and/or further harmonization within the EU, the stability and legitimacy of new social and political institutions should be prime policy targets. Potential benefits of increased well-being include more support for reforms, less emigration, and improved health standards. 


\section{MOTIVATION}

The process of transition in post-communist countries induced considerable and profound changes in political, economic, and social structures and institutions. This so-called "transformational recession" was characterized by rising inequality, unemployment, mortality, and a significant drop in GDP and life satisfaction levels. Economic recovery, however, has been faster than the recovery of the "human costs" of transition, which have ranged from a fall in life expectancy to lower subjective levels of well-being and happiness. A happiness gap-or more generally a "subjective well-being" (SWB) gap-which is the difference in average happiness levels between transition and other countries (usually either non-transition or advanced countries), has led experts to speak of an "iron curtain of unhappiness." Evidence shows that the gap, though gradually closing, still persists.

The amount of information on SWB in different countries is steadily growing. While SWB is used as a general term in the literature, the indicators collected on SWB are usually referred to as "happiness" and "life satisfaction." Happiness mostly encapsulates the emotional-state component of SWB, while life satisfaction is rooted in a reflective judgment and depends mainly on prevailing economic conditions. Earlier research shows that the choice of an indicator may be crucial for the conclusions drawn. Further, it is specified on a case-by-case basis which type of gap was studied by different authors.

The striking heterogeneity, orvariety, of transition experiences among the post-communist countries provides an excellent case study that can be applicable even to advanced economies in times of economic and political upheaval. Common issues include: the wellbeing consequences of policies and reforms; the speed of adaptation to economic and political shocks; the characteristics of reform supporters; and the relative importance of well-being drivers, across a range of socio-economic settings.

\section{History of subjective well-being (SWB) measures}

The systematic collection of SWB measures has a history of slightly more than half a century. In 1957-1963, one of the first data collections purposefully targeting information on "hopes, fears and happiness" was organized, with national samples of 500-2,300 respondents in 14 nations. Later, the introduction of a measure of subjective satisfaction with household income to the newly established German Socio-Economic Panel in 1984 (in particular, for eastern Germany since 1990) and of a set of SWB indicators in the British Household Panel Survey in the 1990s, opened the era of micro-level modeling of well-being and its determinants. The World Values Survey, a multinational data set, has been collected since 1981-1984 (1989-1993 for most transition countries).

The 2013 OECD Guidelines For Measuring Subjective Well-being define it as encompassing three different aspects: (i) a cognitive evaluation of one's life; (ii) positive emotions (e.g. joy, pride); and (iii) negative emotions (e.g. pain, anger, worry). The life evaluation component captures an individual's personal experience and evaluation of life as a whole. This includes general life satisfaction based on income, health, family life, and work life. More recently, the so-called "eudemonic" considerations, i.e. measures of the "meaningfulness" or "purpose" of life, have been added to the measures of interest. A range of positive and negative emotional states and feelings, often referred to as "affects," can be experienced contemporaneously and typically measured with reference to a particular point in time. 
Understanding the causes and consequences of the happiness gap, and learning how best to manage it, can help to close the gap between the new and old EU members and to contribute to the harmonization process within the EU.

\section{DISCUSSION OF PROS AND CONS}

\section{The concept of "well-being"}

Until several decades ago, the academic concept of well-being in economic sciences was represented by a range of objective measures, such as GDP per capita and life expectancy [2]. Such indicators, however, were subsequently considered to be misleading when trying to assess how a population experiences social and economic progress and conditions in a country. A new strand of literature emerged in the 1970s that was dedicated to SWB. Since then, a number of studies have demonstrated that the strength of the association between income and (reported) happiness changes depending on the perspective taken, i.e. the type of indicator (happiness or life satisfaction) and the type of data used (e.g. trends, cross-sectional, or panels). One pioneering study proposed that, within each country, the higher economic status groups were happier than the lower status groups, though paradoxically, GDP per capita levels and its growth over time did not mirror the level and growth of average happiness level [2].

Today, SWB measures are recognized in many countries as being a reliable way of assessing living conditions from a perspective that is different from the traditional economic view [2]. A well-being approach allows the possibility of evaluating the direct and indirect consequences of social and economic reforms and shocks, and helps to disentangle the various contributory elements-such as political circumstances-that might otherwise have been overlooked due to their relatively low impact on life satisfaction in stable economies. In countries undergoing transition, however, the political climate may well have more impact on well-being than the prevailing economic conditions.

Levels of life satisfaction vary significantly between countries around the world (Figure 1). When estimating SWB gaps, most often either a group of non-transition or only advanced economies serve as their counterpart. While the former comparison group helps to assess the general well-being standing of a transition country, the latter would be more appropriate to evaluate how close a transition economy is to an advanced country that was chosen as the target of the transformation process. What should also be kept in mind, however, are the huge differences between post-communist economies. For example, the magnitude of the happiness gap in comparison to advanced economies is likely to be different for the Baltic states relative to the Central Asian states.

\section{Traces of the gap under communist rule}

In the early 1960s, European communist countries ranked in the middle of the happiness scale [3]. The lowest position (at 1.6) on the "11-step ladder" happiness scale $(0=$ "completely unhappy" to 10 = "completely happy") was taken by the Dominican Republic (which was undergoing a period of political instability and armed intervention), while the highest position (at 6.9) was taken by the US (during a period of economic expansion). Three countries of interest-Slovenia (5.3), Croatia (4.8), and Poland (4.4)-were still 


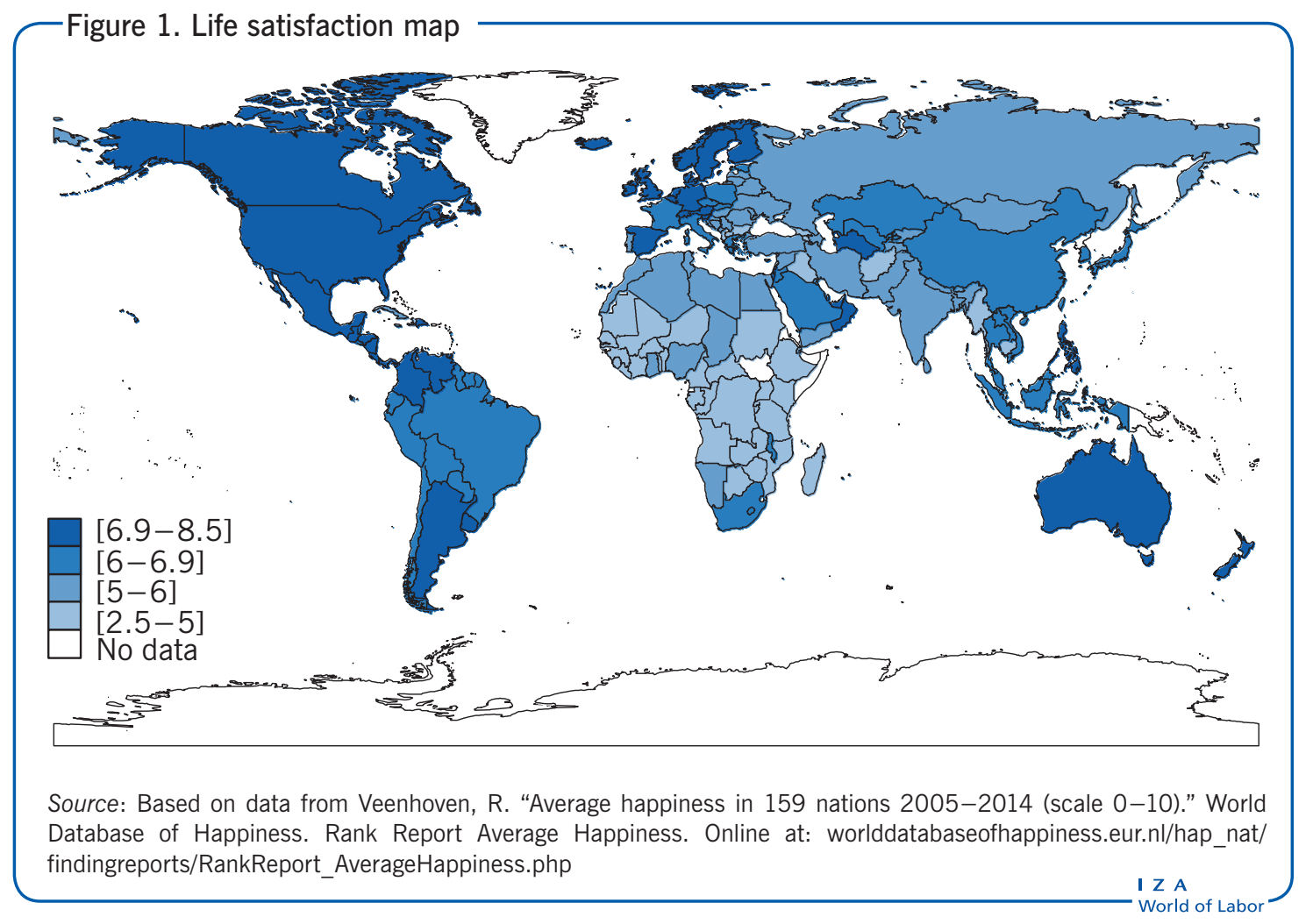

struggling through the post-World War II recovery; their position was similar to that of Brazil (4.7; stagnation in the industrial sector due to unfavorable macroeconomic conditions), Nigeria (4.79; right after officially recognized independence), Japan (5.20; rebuilding industrial capacity and gaining the momentum of economic growth), and Egypt (5.45; on the process of industrialization).

Though these estimates are not directly comparable, due to the different periods and populations covered, they nevertheless give a rough approximation of the SWB gap. With the population sizes of each country in 1960 taken as weights, the weighted mean of happiness in transition countries can be estimated at 4.5 , which is only 0.2 points lower than the average happiness of the non-transition countries in the study [3]. However, in comparison to single countries such as (western) Germany and Japan the gap widens to almost one step of the ladder, and even to 2.5 steps compared to the US. These evaluations, however, even though they support the evidence of a happiness differential at the beginning of the 1960s, should be viewed with caution.

By the beginning of the 1980s, while the official ideology claimed that almost every member of the socialist society was satisfied [4], the signs of the well-being "malaise" were well pronounced. Low levels of SWB accompanied other manifestations of stagnation and demoralization, such as growing alcohol consumption, absenteeism from work, and decreasing male life expectancy [4], [5]. The depth of the well-being gap seemed to correlate with two factors: (i) length of exposure to the communist ideology; and (ii) the extent of application of the communist rule principles.

During the 1980s, well-being indicators deteriorated further. The percentage of "happy" (78\%) and "satisfied" (71\%) Hungarians in 1981 diminished to 68\% and 56\%, respectively, 
in 1990. A particularly dramatic slump of SWB was documented for Russians-as well as for Belorussians, Ukrainians, Bulgarians, and Romanians-providing evidence of the lowest levels ever recorded by 1990 [6]. Immediately prior to the dissolution of the Soviet Union, the shares of "unhappy" populations in five regions of the country were notable: 48\% in Moscow; 52\% in Lithuania; 55\% in Latvia; 44\% in Estonia; and 55\% in Belarus. Contemporaneously, only $17 \%$ of western Germans and $10 \%$ of Americans reported being "(very) unhappy." That sharp decline in SWB among the communist countries seemed to pre-announce the upcoming dramatic changes.

\section{Development of the happiness gap since the early 1990s}

The fall of the Berlin Wall in 1989 triggered the social and economic transition and liberation from communist rule in Central and Eastern Europe (CEE) and, following the Soviet Union's dissolution in 1991, in its former 15 republics. This transition is the only transformation that has taken place without extensive violence, and astonishingly quickly. Significant success in CEE countries led to the enlargement of the EU, and Russia was recognized as a market economy in 2002.

However, the bright story of success also has its dark side. During the first period of transition (circa 1989-1994), major social and economic reforms were implemented alongside macroeconomic austerity measures. By the mid-1990s, when GDP sank to levels of 50-85\% (in comparison to 1989), euphoria and hope of a fast and painless transition from central planning to a market economy evaporated. This also showed in SWB measures, which decreased to below pre-reform levels during the transitional recession, and to below levels of non-transition countries with similar per capita GDP. Russia had beaten its own drastic unhappiness record established prior to the dissolution of the Soviet Union. Recalling the economic and societal differences among countries, the conditional satisfaction gaps (accounting for country characteristics and GDP levels) were estimated: while the life satisfaction of transition countries approximated to 4.7, the gap lay in the range of 1.4 to 1.8 points on a 1-10 scale [1], [7]. The magnitude of the gap was nearly as big as the one dividing US citizens at the 75th and 25th income percentiles [1].

Economic and subjective well-being recoveries in CEE both began in the mid-1990s. During the period 1995-2007, these countries belonged to the fastest-growing economies in the world. The recovery was much slower in the countries of the former Soviet Union (FSU) [8]. The phrase "iron curtain of unhappiness" was coined to describe the persistently lower levels of SWB in transition versus non-transition countries [4]. During 1994-2004, the "raw," or unconditional, SWB gap (i.e. not controlling for a range of other economic, social, and political considerations in the country) shrank by only $20 \%$, or by 0.27 points to 1.13 on the 1-10 scale [1]. Interestingly, the 20 years of transition had a more pronounced negative impact on the older generations. While in advanced countries a U-shape of life satisfaction over the life span is observed, in CEE and especially in the FSU states, a uniform decline in the age-life satisfaction pattern emerged. Due to the absence of the $\mathrm{U}$-shaped relation between SWB/life satisfaction and age in transition countries, the raw satisfaction gap between these two groups of countries increases further with age. It becomes evident when comparing the 7.8 satisfaction score of an average American at 20 -decreasing to 6.8 in the late $30 \mathrm{~s}$, and increasing back to 7.8 at the age of 60 -with 
their transition country counterparts. This is several points higher than 5.99 and 4.3 at 20 and 60, respectively, for an average Russian, and 7.0 and 4.95, respectively, for an average Hungarian in 2006, according to the Gallup World Poll [4].

Today, a raw satisfaction gap between transition and advanced countries of about 0.9 points on the 11-step scale can still be observed (Figure 1). However, the most recent results show advanced economies at the moment that their growth rates were hit by the Great Recession, while some of the transition countries were not yet fully impacted by the economic crisis [1]. Therefore, because the raw gap estimates do not account for the societal and political differences between the two groups of countries, the factors that might potentially lead to the narrowing of the gap in the future, in both situations of economic growth and recession, need to be further investigated.

\section{Drivers of differences in life satisfaction between countries}

Human hopes and concerns, and hence life satisfaction, are formed by a range of factors: economic conditions, health, family, personal values, job and work situation, the international situation, social values, and the political situation [3]. The relative importance of these factors varies both within and across countries. The happiness literature underlines income, family, and health to be among the most referred to determinants of life satisfaction, while political circumstances are among the least quoted among the populations of advanced economies [2].

\section{Economic concerns}

In transition countries, material concerns became pivotal in the first five years of reforms, which had witnessed dramatic falls in output and GDP, in combination with hyper-inflation and soaring unemployment figures. Thus, unlike advanced economies, where there appears to be no link between GDP trends and SWB under stable economic conditions [2], in transition countries higher income (at individual, family, regional, and country levels) and income growth did increase life satisfaction. Such extreme sensitivity to income changes (i.e. growth as well as decline) suggested that further economic growth would also lead to higher well-being levels in post-communist countries. However, SWB mirrored the "U-shaped" GDP trend in only 11 of the 23 transition countries [7]. Six countries (Azerbaijan, Bosnia and Herzegovina, Georgia, Hungary, Macedonia, and Serbia and Montenegro) experienced a fall in life satisfaction, despite economic progress.

While satisfaction with material living levels was growing in the majority of transition countries, the satisfaction with work, family life, and health was decreasing at an individual level [2]. The latter may be connected to falling life expectancy until the mid2000s, especially in the FSU area. However, the dynamics of life expectancy played little role in explaining the national satisfaction levels [5].

\section{Work concerns}

While the benefits of economic growth on SWB seem to diminish when the population adapts to its new level, the same cannot be said of unemployment. In the mid-1990s one study concluded that in rich countries, policies aimed at fostering further economic growth would be of little value. Instead the author of the study suggests to focus on 
employment as a powerful source of happiness. The same could also be true for transition economies.

Many studies in advanced economies reveal a permanent, rather than temporary, detrimental impact of unemployment on SWB, which was also reconfirmed for transition economies between 1995 and 1997, with the exception of Azerbaijan. Thus, unemployment (which is synonymous with uncertainty around the world) was one of the main factors influencing SWB during the first decade of transformation. The detrimental impact of unemployment status on life satisfaction in transition economies was aggravated by two considerations, namely the shock of being unemployed in the first place (which was previously unknown), alongside the drastic social stigma inherited from communist times.

The relatively high predictability (and hence certainty) of the economic and social environment under communist rule might explain why the massive liberalization reforms at the beginning of the 1990s turned the "planned" lives of people upside down. Suddenly, stable prices, low economic and occupation mobility, and guaranteed employment became things of the past. Especially at the beginning of the reforms, when further prospects became unclear and adaptation to the new reality had just started, uncertainty directly and indirectly depressed SWB levels in transition countries.

Adding to this, unemployment benefits were virtually non-existent at the beginning of transition, while the occupational structure was drastically changing. In communist times, the stigma of social disapproval was attached to not working. In the Soviet Union, belonging to the bottom $20 \%$ of the income distribution would induce shame of being poor and hence had a strong negative effect on SWB. As a salary was the main-and often the only-source of income for the majority of FSU citizens, unemployment meant being declassified into the category of being poor. These issues imposed a double psychological burden: of not being a productive member of society and of being poor.

For the employed in transition countries, however, satisfaction with jobs was both among the lowest of different life domains and lower than in Western European countries. One explanation for this is a general lack of commitment to work in post-communist countries, as a result of work being perceived simultaneously as a right and an obligation. Until at least the early 2000s, Western European workers placed a higher weight on the internal aspects of a job (e.g. sense of responsibility, social interaction), while workers from transition countries valued more the external aspects (e.g. pay, holidays) [9]. Meanwhile, real wages dropped to $40-90 \%$ of 1989 levels across post-communist countries [2]. Thus employment itself, especially through the 1990s, had limited power in reducing the risk of poverty and individual uncertainty. From the advanced economies it is known that a high level of job insecurity is comparable to unemployment in terms of its detrimental impact on SWB. Yet a (permanent) job with wage arrears was still preferred to unemployment in the Russia of the 1990s, possibly due to the hope of receiving wages outstanding at a later time, or as a result of the benefits accessible by employees. To compensate for the psychological/SWB costs of wage arrears one would require a payment equal to twice the household income.

\section{Social and institutional concerns}

As for the labor market institutions that might have mitigated the detrimental impact of uncertainty, their role and coverage also changed significantly. Membership of trade 
unions was transformed from forced to voluntary, and their role progressed from the provision of social duties indirectly connected to jobs (e.g. organizing holiday trips) to collective bargaining structures. Membership coverage was also significantly reduced in most of the transition countries. Attitudes toward extensive state interventions, and to the state as the main provider of jobs, have been fading at a slower pace.

The introduction of new institutions has proven to be a long and difficult process. The successful development of democratic institutions, and the improved extent of political and civil liberties in CEE has been well documented [7]. Nevertheless, evidence for the FSU suggests the creation of a "legislation vacuum" during the first stage of reforms and a misinterpretation of democracy as doing whatever one wants, rather than what the law permits. Across many post-communist states during the 1990s, this gave rise to the accumulation of capital and the establishment of "new rules" by a group of high-level, ex-Communist Party functionaries and a growing cadre of elite individuals, who managed to accumulate wealth by circumventing the law [10].

In most of the transition countries, reforms began with the liberalization of prices, trade, and foreign exchange, and the privatization of small businesses. Large-scale privatization was launched later and is still to be completed in the Western Balkans and the Commonwealth of Independent States. While the earlier reforms improved individual perceptions of the transition, and hence SWB, the large-scale privatization process had the opposite effect, in that it solidified the popular view of business and political leadership benefiting from the reforms and, as a result, pushed down the SWB of people [9]. Perceived lawlessness, corruption, bad governance, and low legitimacy of the new institutions are still the characteristics of some transition countries; yet these factors have an unclear impact on SWB in the post-communist region [7], [11]. Thus, the perception of corruption makes a difference for life satisfaction in inter-country comparisons, but not at the personal level, as individuals have personal views on the extent of bribery and corruption [10]. There is a belief in merit leading to success and good evaluation of governmental performance that makes individuals happier. Recent research also suggests that the development of "a system of well-defined, universally applicable, and fair laws" has the power to enhance life satisfaction in general and to close the SWB gap between the transition and advanced economies in particular" [1]. The magnitude of the system's general positive impact on SWB, however, is twice as great for advanced economies as for non-EU transition ones.

Besides the establishment of the new political and economic institutions, an equally painful process of social disintegration took place in post-communist countries. The conflict between the old and the new realities led to social instability and unpredictability of the social order. The latter is also referred as "social anomie," namely a low consensus on norms, a lack of clarity on societal values, and strong feelings of injustice. In some cases, e.g. Russia, a huge drop in the sense of national self-esteem was observed [6]. A "spiritual vacuum" appeared, which led to the substitution of communist ideology by religious practices [10]. Religion has been shown to insure happiness against negative effects of reforms in transitional countries [12]. However, the impact of religion is controversial. Higher shares of the Eastern Orthodox religion are found to be correlated with weaker economic growth and with lower life satisfaction in country-level relationships [10].

In addition, regardless of the flourishing of religion, a "culture of distrust" spread across Eastern European societies, inducing yet another gap between transition and advanced 
economies: the "social capital" gap [7]. Recent literature connects higher levels of social trust with a safer environment, higher social cohesion, and good governance. Aggregate social trust has been proven to be a strong predictor of SWB, even in the most extreme cases-such as transition countries during periods when economic performance is weak [11].

\section{LIMITATIONS AND GAPS}

Evaluating the significance and magnitude of happiness gaps involves several potential drawbacks. First, SWB data may not be internationally comparable. Thus, the discussion on whether cultural differences in sharing feelings and concerns are responsible for the persistence of international SWB gaps is not yet complete. A test of comparability of the results across several age groups in Russia and the US, for instance, led to mixed conclusions. While responses of students were comparable, the elderly were found to be more prone to share and identify with others' misfortune in Russia than in the US. Moreover, the Russian elderly tended to idealize the pre-reform period and hence reported a bigger drop in SWB and living standards than was perhaps the case in reality [13]. This result warns of a potential measurement bias with respect to culture and intergenerational differences. Thus, the demographic structure of the population should be taken into account when making international comparisons.

A second issue is the relatively low quality of data for transition countries. In the widely used World Values Survey (WVS), post-communist countries' samples are often overpopulated by low-income households. Thus an improvement of sample quality is advocated to potentially close the SWB gap by 0.33 points [7]. Other sources warn that the WVS includes only a relatively small number of poor countries. While Eastern European and FSU economies are categorized as "poor," especially in the earlier years, the income estimates are among the most unreliable. In the case of the poorest economies, samples cover mostly literate and urbanized populations, which are often happier, and hence another source of bias for the gap estimation is introduced [4].

Third, due to the persistently poor data coverage of the transition countries, and only fragmented coverage of advanced/non-transition economies in the past (pre-1990s), the sets of countries labeled "non-transition" and "transition" may differ significantly across years. To compensate for the data unavailability, several sources of information (i.e. various surveys) might be exploited contemporaneously. This however leads to the question of the comparability of the wording of SWB questions and the scaling of answers. Standardization of responses to make them comparable across countries may be sensitive to the proportion of happy and unhappy countries, and cultural differences, in the data sets [10].

\section{SUMMARY AND POLICY ADVICE}

The 25 years since the fall of the Berlin Wall have shown that transition is an unhappy process associated with huge losses in terms of health and happiness-despite economic development and improvement of political and civil liberties, including the establishment of democracy in post-communist societies. As economic conditions between transition and non-transition groups were converging, the SWB gap was gradually narrowing, though at a slower speed. 
Recent literature shows both the persistence of the happiness/life satisfaction gap as well as evidence for the absence of a gap in the aftermath of the economic crisis of 2008. The latter could be taken as a sign of the transition period being completed, at least in the minds of the citizens. However, before giving credence to one or another opinion, consideration should be given to the heterogeneity of the post-communist countries, in terms of economic and institutional progress, and to the choice of the comparison groups (advanced, non-transition countries). In such a framework, the transition success of the new European member states becomes even more evident.

The experience of transition countries demonstrates that in times of economic and political upheaval, uncertainty at the national and individual level not only takes a heavy toll on SWB, but can also modify the impact of different conditions and factors on SWB. Whilst it is true that economic growth and improved living standards in the "catching-up" economies may further reduce the international gaps in living standards, and hence in happiness, there are other issues to consider. First, economic progress is not guaranteed, as the example of Ukraine shows, and second, a strategy of reducing the unemployment figures and general economic volatility, and for enhancing social, political, and economic stability and governmental effectiveness, has the potential to bear fruit more rapidly and have a positive impact on SWB, over a longer time span.

While new institutions can be introduced quickly, it is clear that economic and institutional reforms can burden the population with short-, medium-, and long-term well-being costs. In order to reduce the time taken to adapt to the new rules (and hence the time it takes to reduce the SWB gap), policymakers should provide the population with clear information on the short- and long-term consequences of the reforms, as well as with detailed (but easily understandable) reports throughout the period of a policy/reform implementation.

Increased transparency, coherence, and legitimacy of reforms, the universal applicability of laws, and lower corruption levels are all factors than can improve well-being. Higher life satisfaction of the population, in its turn, lowers the probability of reform fatigue and prevents a "reform backlash." Thus, ensuring the stability of happiness levels may contribute significantly to enhancing the stability of newly formed institutions.

\section{Acknowledgments}

The author thanks two anonymous referees and the IZA World of Labor editors for many helpful suggestions on earlier drafts.

\section{Competing interests}

The IZA World of Labor project is committed to the IZA Guiding Principles of Research Integrity. The author declares to have observed these principles.

(c) Ekaterina Skoglund 


\section{REFERENCES \\ Further reading}

Hölschner, J,. and H. Tomann (eds). Palgrave Dictionary on Emerging Markets and Transition Economics. Basingstoke: Palgrave Macmillan, 2016.

Selezneva, E. "Surveying transitional experience and subjective well-being: Income, work, family." Economic Systems 35:2 (2011): 139-157.

\section{Key references}

[1] Nikolova, M. Minding the Happiness Gap: Political Institutions and Perceived Quality of Life in Transition. IZA Discussion Paper No. 9484, 2015.

[2] Easterlin, R. A. "Lost in transition: Life satisfaction on the road to capitalism." Journal of Economic Behavior \& Organization 71:2 (2009): 130-145.

[3] Cantril, H. Pattern of Human Concerns. New Brunswick, NJ: Rutgers University Press, 1965.

[4] Lelkes, O. "Tasting freedom: Happiness, religion and economic transition." Journal of Economic Behaviour \& Organization 59:2 (2006): 173-194.

[5] Deaton, A. "Income, health and well-being around the world: Evidence from the Gallup World Poll." The Journal of Economic Perspectives 22:2 (2008): 53-72.

[6] Inglehart, R., R. Foa, E. Ponarin, and C. Welzel. Understanding the Russian Malaise: The Collapse and Recovery of Subjective Well-being in Post-communist Russia. Moscow National Research University Higher School of Economics Working Papers BRP 32/SOC/2013, 2013.

[7] Guriev, S., and E. Zhuravskaya. "(Un)happiness in Transition.” Journal of Economic Perspectives 23:2 (2009): 143-168.

[8] Grün, C., and S. Klasen. "Has transition improved well-being?” Economic Systems 36:1 (2012): 11-30.

[9] Borooah, V. K. "Comparing levels of job satisfaction in the countries of Western and Eastern Europe." International Journal of Manpower 30:4 (2009): 304-325.

[10] Djankov, S., E. Nikolova, and J. Zilinsky. "The happiness gap in Eastern Europe." Journal of Comparative Economics 44:1 (2016): 108-124.

[11] Bartolini, S., M. Mikucka, and F. Sarracino. "Money, trust and happiness in transition countries: Evidence from time series.” Social Indicators Research 130:1 (2017): 87-106.

[12] Popova, O. "Can religion insure against aggregate shocks to happiness? The case of transition countries." Journal of Comparative Economics 42:3 (2014): 804-818.

[13] Tucker, K. et al. "Testing for measurement invariance in the satisfaction with life scale: A comparison of Russians and North Americans." Social Indicators Research 78:2 (2006): 341-360.

\section{Online extras}

The full reference list for this article is available from:

http://wol.iza.org/articles/the-happiness-gap-between-transition-and-non-transition-countries

View the evidence map for this article:

http://wol.iza.org/articles/the-happiness-gap-between-transition-and-non-transition-countries/map 